\title{
Pertinencia del uso de implantes dentales cortos en pacientes con atrofia ósea severa. Revisión de la literatura
}

\author{
Relevance of use of short dental implants in patients with severe bone \\ atrophy. Literature review
}

\author{
AZAÑ̃́N HERNÁNDEZ R* \\ MARTÍNEZ LARA I** \\ FERRER GALLEGO $\mathrm{J} * * *$ \\ MARZO ALZOTA $\mathbf{R} * * * *$
}

Azañón Hernández R, Martínez Lara I, Ferrer Gallego J, Marzo Alzota R. Pertinencia del uso de implantes dentales cortos en pacientes con atrofia ósea severa. Revisión de la literatura. Av Periodon Implantol. 2013; 25, 3: 153-164.

\section{RESUMEN}

El propósito de este artículo es determinar la pertinencia del uso de implantes cortos, definiéndolos como "aquellos cuya longitud es $\leq 8 \mathrm{~mm}$ " a través de la bibliografía existente. Hemos centrado la búsqueda en la comparación del uso de implantes de esta longitud, frente a otros tratamientos alternativos (injertos óseos, elevación de seno, transposición del nervio dentario, etc.) en pacientes con atrofia maxilar severa.

Se dan respuesta a las siguientes cuestiones: ¿El uso de implantes dentales cortos es un tratamiento de resultados previsibles? ¿Los porcentajes de éxito a medio y largo plazo son equiparables a los de implantes con una longitud media estándar? ¿Pueden sustituir en determinadas situaciones clínicas a técnicas quirúrgicas avanzadas (injertos óseos, elevación de seno, distalización del nervio dentario) disminuyendo con ello la morbilidad, los tiempos en la rehabilitación y los costes para el paciente? ¿Se requiere de un protocolo clínico y prostético específico para garantizar el éxito en la rehabilitación? ¿En qué casos de atrofia maxilar se contraindica esta técnica a favor de otras como son los implantes cigomáticos o los injertos óseos? A través de una búsqueda cuasi-sistemática en metabuscadores, agencias de evidencias (revisiones sistemáticas) y bases de datos bibliográficos, exponemos la evolución de la evidencia al respecto, los últimos datos publicados y las conclusiones obtenidas.

PALABRAS CLAVE: MeSH (Medical Subject Headings), implantes dentales, implantación dental, endoóseo, pérdida de hueso alveolar/rehabilitación, pérdida de hueso alveolar/cirugía, implantes dentales largos y cortos.

\section{SUMMARY}

The purpose of this review is to determine the relevance of the use of short implants, defining them as "those whose length is $\leq 8 \mathrm{~mm}$ " according to the existing bibliography about it. We have centred the search on the use of implants of this length in comparison to alternative treatments (bony grafts, bone augmentation, dental nerve transposition, etc.) in patients with severe maxillary atrophy. It provides answers to the following questions: Is the use of short dental implants a treatment of foreseeable results? Are the percentages of success in the medium and long term comparable to

\footnotetext{
* Licenciado en Odontología. Máster Universitario en Cirugía Oral, Implantología y Periodoncia. Clínica Dental Azañón. Málaga.

** Licenciado en Medicina. Especialidad en Cirugía Maxilofacial. Profesor Asociado de la Facultad de Ciencias de la Salud del Departamento de Estomatología. Universidad de Granada.

*** Licenciado en Odontología. Clínica Dental Ferrer Gallego. Granada.

**** Licenciado en Odontología. Clínica Dental Marzo. Vizcaya.
} 
those of implants with an average standard length? In certain clinical situations, can they be used as a substitute for other advanced surgical techniques (bony grafts, bone augmentation, distalization of the dental nerve), diminishing with it the morbidity, the rehabilitation periods and the cost for the patient? Is it required a specific prosthetic and clinical protocol to guarantee the success in the rehabilitation? In which cases of maxillary atrophy is this technique/treatment contraindicated in favour of other ones like zygomatic implants or bone grafts? Through an almost-systematic search in meta-search engine, agencies of evidence (systematic reviews) and bibliographical databases, we expose the evolution of the evidence on the matter, the most recent data published and the conclusions obtained.

KEYWORDS: MeSH (Medical Subject Headings), dental implants, dental implantation, endosseous, alveolar bone loss/rehabilitation, alveolar bone loss/surgery, length and short dental implants.

Fecha de recepción: 15 de febrero de 2012.

Fecha de aceptación: 20 de marzo de 2012.

\section{INTRODUCCIÓN}

Los implantes dentales han revolucionado la Odontología contemporánea. Desde que el concepto de oseointegración fue introducido en 1969, la ciencia y la tecnología de los implantes dentales han experimentado una evolución exponencial. La previsibilidad y la eficacia de los implantes dentales en la rehabilitación de pacientes con edentulismo total y parcial, ha sido demostrada por completo en este tiempo. Como resultado de ello, se extendió su uso e indicación, mejorando la tasa de éxito desde el $85 \%$ en los años ochenta a casi el $99 \%$ actualmente. El edentulismo conlleva fundamentalmente, atrofia vertical en la mandíbula y reabsorción centrípeta (horizontal) más neumatización de los senos paranasales en el maxilar superior. Actualmente es un tema de notorio interés, debido a que un considerable número de los pacientes subsidiarios de implantes dentales, sufren algún tipo de atrofia maxilar. El edentulismo parcial de mandíbula posterior es una situación clínica bastante común. La prótesis parcial removible es mal tolerada debido a la inestabilidad de la misma por acción de la lengua y la molestia que ello supone para el paciente. La solución ideal es la colocación de implantes que soporten una prótesis fija, con una longitud que oscile al menos entre los 9 y los $12 \mathrm{~mm}$ y que puedan garantizar un pronóstico bueno a largo plazo. Desafortunadamente, a menudo, los pacientes edéntulos, presentan una altura de cresta mandibular insuficiente que hace necesario la colocación de injertos óseos. Es frecuente encontrar una altura menor a $10 \mathrm{~mm}$, por lo que el uso de prótesis soportadas sobre implantes cortos, podría ser considerado un tratamiento con mayor riesgo de fracaso (según algunos autores) aunque puede ser una alternativa más rápida, más sencilla y más barata que los procedimientos de injerto óseo (8). Es por ello, por lo que surgen nuevas preguntas en la búsqueda de la mejora si cabe, en el uso de una u otra técnica; teniendo como alternativa entre otras, el uso de implantes con longitud más corta que la estándar utilizada hasta ahora, para abrir la posibilidad de colocación de los mismos a un mayor número de pacientes, obviando el recurrir a técnicas quirúrgicas avanzadas con todo lo que ello conlleva. Los estudios sobre los diferentes tipos de implantes, sobre todo se centran en determinar aspectos globales que nos hagan inclinarnos hacia uno u otro modelo de implante, pero pocos son los ensayos clínicos aleatorizados o metaanálisis que establezcan los implantes dentales cortos como alternativa terapéutica a cualquiera de las otras opciones para el tratamiento de la atrofia ósea. Esto supone un problema a la hora de buscar artículos que nos ayuden a tomar una decisión clínica, ya que para comparar adecuadamente el efecto de las diferentes características de implante, como por ejemplo la longitud del mismo, el ensayo ideal debería ser diseñado de forma que sólo la característica de interés sea diferente (grupo prueba versus grupo control) mientras que el resto de los parámetros deben ser idénticos. Esto no es el caso para la mayoría de los ensayos controlados aleatorios (ECAs), dado que la mayoría de los ensayos encontrados, comparan una combinación de diferentes características de superficie, formas, dimensiones, diferente pureza del titanio y colocados con diferentes protocolos quirúrgicos (sumergido versus no sumergido, carga temprana o inmediata, uso de diferentes tipos de injertos, etc.). En 2009, Marco Espósito y cols. (10) concluyen que: "Diversas técnicas pueden aumentar el hueso en dirección horizontal y vertical, 
pero no está claro cuál es la más eficiente. Los implantes cortos parecen ser una mejor alternativa al injerto óseo vertical del maxilar inferior reabsorbido". Pero hay una gran variación entre los distintos procedimientos de aumento de hueso con diferentes biomateriales, en combinación con los biológicamente activos. Aunque la superioridad de una técnica frente a otra, está continuamente discutida en la bibliografía. El uso de estas técnicas requiere, por un lado, la destreza del clínico en el manejo de las mismas y suponen el aumento del tiempo de espera para realizar la carga protésica.

Hace tan solo 10 años, un plan de tratamiento que contemplase colocar implantes de 6-7 mm era impensable y sinónimo de fracaso garantizado ya que, en la conciencia colectiva del terapeuta (y en los estudios al respecto), se concebía la longitud mínima del mismo como un parámetro irrenunciable para garantizar la estabilidad primaria, la amplitud total de la interfase implante-hueso y proporcionar resistencia a las fuerzas de torsión. Numerosas publicaciones abordan el estudio de la longitud del implante como un pronosticador de supervivencia $(12,45)$. Pero estos trabajos han reportado resultados contradictorios. Algunos estudios informan de porcentajes de fracasos más altos con implantes cortos $(20,42)$ y otros informan de supervivencia alta $(38,39)$. Aquellos estudios que informan las tasas favorables de supervivencia tienden a ser encontrados en publicaciones más recientes, pudiendo indicar que el éxito clínico con implantes cortos, quizás había mejorado durante los últimos años. La justificación en el uso de los mismos, supone un aumento del número de pacientes que pueden beneficiarse de una rehabilitación oral con implantes dentales, reduciendo la morbilidad del procedimiento, acortando los tiempos y disminuyendo el coste del tratamiento. No obstante, cuando hablamos de la longitud del implante como un parámetro a valorar en la mejora de la osteointegración y del éxito terapéutico, hemos de considerar el valor y relevancia de otro parámetro como es el diámetro. Paralelamente a los estudios de implantes denominados "cortos", podríamos hablar también de los implantes de diámetros más pequeños. Son los llamados implantes dentales minis (MDIs). Estos tienen generalmente de entre 2,75-3,30 $\mathrm{mm}$ de diámetro, y son utilizados con frecuencia en casos de volumen limitado de hueso, aunque la principal indicación de MDIs cuando se introdujeron en el mercado, fue la provisionalización (servir como recurso temporal para la colocación de restauraciones provisionales durante la fase de osteointegración de los implantes convencionales). Aunque no es el objeto de nuestro estudio, tomaremos en consideración el parámetro del diámetro ya que aparecen muy vinculados ambos en repetidas ocasiones a lo largo de la revisión realizada.

Por lo tanto, nos preguntamos: ¿El uso de implantes dentales cortos es un tratamiento de resultados previsibles? ¿Los porcentajes de éxito a medio y largo plazo son equiparables a los de implantes con una longitud media estándar? ¿Pueden sustituir en determinadas situaciones clínicas a técnicas quirúrgicas avanzadas (injertos óseos, elevación de seno, distalización del nervio dentario) disminuyendo con ello la morbilidad, los tiempos en la rehabilitación y los costes para el paciente? ¿Se requiere de un protocolo clínico y prostético específico para garantizar el éxito en la rehabilitación? ¿En qué casos de atrofia se contraindica esta técnica a favor de otras como son los implantes cigomáticos o los injertos óseos? En resumen, tras la lectura detallada de este informe, pretendemos exponer al lector lo que hasta ahora la bibliografía publicada nos ofrece, para que el Implantólogo tenga la oportunidad de aplicar tratamientos en base a una "Odontología Basada en la Evidencia" y orientar acerca de hacia dónde debemos dirigir nuestros esfuerzos en materia de investigación futura.

\section{MATERIAL Y MÉTODO}

Se realizó una búsqueda cuasi-sistemática en las bases de datos, utilizando la plataforma OVID, que incluye las siguientes bases de datos:

$\square$ Books@Ovid May 12, 2011.

$\square$ Sumarios y resúmenes todo Ovid.

$\square$ Revistas suscritas por la Biblioteca Virtual del SSPA (Texto Completo).

$\square$ Ovid MEDLINE(R) In-Process \& Other NonIndexed Citations and Ovid MEDLINE(R) 1948 to Present.

$\square$ Ovid MEDLINE(R) Daily Update May 12, 2011.

$\square$ Ovid OLDMEDLINE(R) 1946 to 1965.

$\square$ EMBASE Excerpta Medica Database.

Hemos acotado la búsqueda en revisiones sistemáticas (Cochrane Central Register of Controlled Trials CENTRAL), estudios comparativos, metaanálisis, ensayos clínicos, ensayos controlados aleatorizados identificados (ECAs) y estudios multicéntricos desde 2005 hasta el momento de la revisión. Para la identificación de los estudios incluidos o considerados para esta revisión, se desarrolló una estrategia de búsqueda detallada. 
La estrategia de búsqueda usó una combinación de vocabulario controlado (Mesh) y de términos de texto libre basada en lo siguiente: 1. exp. (Dental Implants/) 2. exp. (Dental Implantation/ or dental implantation, endosseous) 3. \#1 or \#2 and (short) 4 . \#1 or \#2 and (length) 5. \#1 or \#2 and (Alveolar Bone Loss/ rehabilitation* or Alveolar Bone Loss/surgery*) 6. \#5 and (short) 7. \#5 and (length). Se examinaron los títulos y los resúmenes (disponibles) de todos los artículos identificados a través de las búsquedas electrónicas. Se realizó lectura rápida de los artículos encontrados en esta búsqueda. Se obtuvo el artículo a texto completo de los estudios que parecían reunir los criterios de inclusión, o de los cuales había datos insuficientes en el título y el resumen para tomar una decisión clara.

\section{DISCUSIÓN}

\section{Definición de la longitud y el diámetro}

La definición de implantes "cortos" es polémica ya que algunos autores consideran como "corto" aquellos cuya longitud oscila entre los 7 y $10 \mathrm{~mm}$ (8) mientras otros autores consideran "corto", aquellos cuya longitud es $\leq 8 \mathrm{~mm}$ (37). El papel del diámetro del implante es menos conocido, ya que pocos estudios aíslan este parámetro para encontrar la relación directa con la osteointegración y el éxito terapéutico. La mayoría de los clínicos tienden a compensar la escasa longitud de un implante, utilizando un mayor diámetro en el mismo. No resulta fácil hablar de longitud del implante, sin tener en cuenta la influencia del diámetro. Así, Petrie CS y Williams JL (2005) (33) nos recuerdan que el diámetro, la longitud, y la existencia de una cresta alveolar estrecha, tienen que ser considerados conjuntamente, debido a la influencia recíproca de estos tres parámetros sobre el estrés del hueso crestal. Concluyen en su estudio, manifestando que si el objetivo es minimizar el estrés periimplante en el hueso de la cresta alveolar, la elección más favorable es un implante ancho, relativamente largo y cilíndrico (no cónico). Según los autores, los implantes estrechos, cortos y en una cresta delgada, se deben evitar, especialmente en hueso de baja densidad (D3-D4). Volviendo a centrarnos en la longitud del implante, nos basaremos en la definición de "implante corto" que hacen Renouard F y Nisand D. (2006) (37): Aquel cuya longitud intrahueso es $=8 \mathrm{~mm}$. A partir de ésta, establecemos y acotamos nuestra búsqueda bibliográfica. Varios son los autores, que identifican la longitud del implante, como uno de los factores que influyen en el éxito o fracaso de los implantes dentales (35). Según Chung DM, Tae-Ju Oh, Lee J, Misch CE y Hom-Lay Wang. (2007) (7), la longitud del implante fue el factor más significativo en el mantenimiento de los mismos (estabilidad secundaria).

\section{Evolución cronológica de los estudios}

Podríamos agrupar en cuatro principales subgrupos de resultados, los estudios a cerca del uso de implantes cortos respecto de implantes con longitud estándar durante un primer período de tiempo: Algunos artículos mostraron claramente que los implantes cortos fracasaron más a menudo que los largos (Bahat, 1993; Jemt y Lekholm, 1995; Wyatt y Zarb ,1998; Bahat, 2000; Winkler et al, 2000; Naert et al, 2002; Weng et al, 2003; Herrmann et al, 2005, Van Steenberghe D et al). Un segundo grupo, aunque concluyendo que existía un aumento del porcentaje de fallo en implantes cortos, las tasas de supervivencia eran proporcionadas en ambos grupos (Van Steenberghe et al, 1990; Friberg et al, 1991; Jemt, 1991; Lekholm et al. 1999). Un tercer grupo de artículos informó que la longitud del implante no pareció influir aparentemente en la tasa de supervivencia (Buser et al, 1997; Ellegaard et al, 1997; Gunne et al, 1999; Brocard et al, 2000; Testori et al, 2001; Stellingsma et al, 2003; Feldman et al, 2004; Romeo et al, 2004; Lemmerman \& Lemmerman, 2005). Por último, un grupo de artículos enfocados específicamente en implantes cortos indicó resultados de supervivencia semejantes entre éstos y los implantes más largos (88-100\%) (Bernard et al, 1995; Texeira et al, 1997; Ten Bruggenkate et al, 1998; Deporter et al, 2000, 2001; Friberg et al, 2000; Stellingsma et al, 2000; Tawill y Younan, 2003; Fugazzotto et al, 2004; Griffin y Cheung, 2004; Nedir et al, 2004; Goené et al, 2005; Renouard y Nisand, 2005). Los estudios más antiguos, indican que la longitud parece influir poco en la reducción de las tensiones que se producen a nivel de la cresta ósea durante la carga oclusal. Por otro lado, la pérdida temprana del implante, está relacionada con la falta de estabilidad primaria mientras que la pérdida del implante en etapas posteriores, está relacionada con la existencia de infecciones (perimplantitis) y con la sobrecarga funcional (27). El aumento en la longitud del implante mejora la estabilidad primaria y la amplitud total de la interfase entre hueso e implante. Este incremento también proporciona mayor resistencia a las fuerzas de torsión (44).

En la conciencia colectiva de los profesionales dedicados a la Implantología, siempre estuvo presente la 
idea de que a menor densidad del hueso, mayor debiera ser la longitud y el diámetro del implante a colocar. Petrie CS y Williams JL (2005) (33) señalaron que los implantes anchos y relativamente largos constituían la mejor opción de tratamiento. De la misma forma Winkler S, Morris HF y Ochi S (2000) (46) encontraron que implantes cortos y estrechos presentaban un mayor índice de fracaso y una supervivencia menor. En 2003, Pierrisnard L, Renouard F, Renault P y Barquins $\mathrm{M}$ concluyen que los implantes cortos presentan porcentajes de fracaso superiores para todos los casos. (34) Pero en 2004 (21), en la revisión de la literatura en MEDLINE (1985-2001), realizada por Hagi D, Deporter DA, Pilliar RM y Arenovich T, no aparecieron datos concluyentes que identificaran claramente las características en materia de superficie y geometría, que se relacionaran con un menor número de fracasos. Misch (2005) empieza a hablarnos de la necesidad de hacer un uso racional de los implantes cortos, junto con un protocolo específico en estos casos (29).

Nuestra revisión bibliográfica tiene un punto de partida en el 2005, a raíz de la revisión sistemática que Renouard y Nisand realizaron sobre el impacto de la longitud y el diámetro del implante en la supervivencia del mismo (37). Exploraron la relación entre tasas de supervivencia del implante con su longitud y diámetro, en el periodo de 1990-2005, definiendo, entre otros, los criterios para el fracaso. Determinan en su estudio que el uso de implantes cortos o anchos puede ser considerado por el profesional, en ubicaciones donde el éxito de implantes con una longitud estándar es complicado debido a la severa atrofia ósea o a un traumatismo previo. Los resultados pueden ser comparados con procedimientos quirúrgicos avanzados como el injerto de hueso, elevación de seno y la transposición del nervio alveolar. En la revisión presente, un implante "corto" fue definido como un dispositivo con una longitud intraósea $\leq 8 \mathrm{~mm}$, un implante "ancho" aquel cuyo diámetro es $\geq 4,5 \mathrm{~mm}$ e implante "estrecho" $\leq 3,5 \mathrm{~mm}$. Concluyeron que las tasas de supervivencia de los implantes cortos eran comparables con las obtenidas con implantes más largos colocados en condiciones similares, utilizando técnicas quirúrgicas adecuadas e implantes con superficie rugosa. Entre las conclusiones también obtenidas, los autores encuentran una debilidad científica, en tanto en cuanto existe un número pobre de ensayos clínicos multicéntricos y aleatorizados o metaanálisis. Según algunos autores, los implantes largos consiguen una mayor estabilidad primaria y un aumento en la transmisión de fuerzas de un $7,3 \%$ respecto a los implantes cortos $(8 \mathrm{~mm})(24)$, sobre todo en base a los estudios de O'Sullivan D,
Sennerby L y Meredith N (2000) (32), ya que observaron que implantes cortos de diámetro ancho, y colocados en determinado tipo de hueso, presentaban un alto porcentaje de fracaso por aterrajar el lecho óseo del implante. Pero, sin embargo, Goene et al (2005) (19) informó de mejores resultados con implantes cortos de superficie tratada mediante lavado ácido. También en 2005 (23) se empezaron a relacionar los fracasos de los implantes cortos, con el área del maxilar donde eran emplazados. Así concluyeron en una revisión sistemática realizada por Jae-Hoon Lee, Val Frias, KeunWoo Lee y Robert FWright. Con respecto a los implantes cigomáticos, tal y como nos indica Espósito M, Worthington HV y Coulthard P (11B), entre las indicaciones de su uso (declinando utilizar injerto óseo), podemos encontrarnos con dos situaciones clínicas. Una, en la que podamos colocar al menos dos implantes cortos en el maxilar anterior y un cigomático en cada cuadrante o, bien, cuando la atrofia maxilar anterior es tan severa que impide implantar a ese nivel, se podría rehabilitar con dos o tres implantes cigomáticos a cada lado. Sin recurrir a las técnicas de injerto óseo, no hemos encontrado en la bibliografía evidencia acerca de cual es la cresta residual mínima necesaria para la colocación de implantes cortos versus implantes cigomáticos.

Das Neves FD, Fones D, Bernardes SR, do Prado CJ y Neto AJ, en 2006, concluyen que el implante corto ( $\leq 7$ $\mathrm{mm}$ ) tiene peor pronóstico a largo plazo, comparado con implantes más largos, sin embargo, pueden ser una alternativa más rápida, más sencilla y más barata que los procedimientos de injerto óseo (8). En este estudio longitudinal bien diseñado, de información recogida en Medline desde 1980 a 2004, nos refiere que los implantes cortos deben ser considerados como una alternativa a cirugías avanzadas de aumento de hueso, ya que este tipo de tratamientos pueden implicar un incremento de la morbilidad, requieren de períodos clínicos más prolongados e implican un coste mayor para el paciente. Asimismo, reseñan la necesidad de estudios de mayor rigor científico. En una comparación de utilización combinada de implante corto y elevación de seno (Ferrigno N, Laureti M y Fanali S, 2006) (17), los más cortos (8 mm) no fallaron apreciablemente más que los largos (10-12 mm), aunque ninguna conclusión estadística pudo ser extraída, desde el punto de vista clínico, el uso previsible de implantes cortos en conjunción con elevación de suelo de seno maxilar mediante la técnica atraumática (con osteótomos u otra técnica) puede reducir la indicación de procedimientos invasivos complejos como la elevación de seno mediante técnica traumática (de Tatum) 
y los procedimientos de injerto óseo. Misch et al (2006) (29) continúa estudiando y abogando por el uso de los implantes cortos, cuando en este estudio de series de casos, cuyo propósito fue evaluar la supervivencia de estos cuando se elabora un plan de tratamiento con un enfoque biomecánico apropiado, concluye que el uso de implantes cortos es un tratamiento de resultados previsibles en el edentulismo parcial posterior, si se rehabilita mediante prótesis fija y tratando de disminuir el estrés en la interfase hueso-implante (ferulización de implantes, no cantiléver etc.). Anitua E, Orive G, Aguirre JJ y Andrea I (1) (2008), en su estudio, evaluaron la influencia de la longitud, del diámetro y de la geometría de los implantes dentales del Biotechnology Institute en la distribución del estrés óseo. Los resultados mostraron que el efecto del diámetro del implante en la distribución del stress óseo fue más significativo que el efecto de la longitud del implante. Adicionalmente apunta al uso de implantes cortos y más anchos como alternativa razonable en emplazamientos con limitación de la altura en la cresta residual. En 2008, empiezan a aparecer estudios que combinan el uso de implantes cortos y técnicas de demostrada solidez, como es la carga inmediata. Parece quedar demostrado que también se puede utilizar carga inmediata con implantes de $7 \mathrm{~mm}$ de longitud, tal y como presentan en este estudio randomizado, Cannizzaro G, Leone $M$, Torchio C, Viola P y Espósito M (6); aunque también indican que es importante hacer un seguimiento a largo plazo para evaluar la efectividad del tratamiento instaurado con el paso de los años.

En 2009, este mismo grupo de trabajo acepta el hecho de que se utilizan implantes cuya longitud oscila entre 5-8 $\mathrm{mm}$, pero pocos son los estudios que evalúan en profundidad, su eficacia como tratamiento alternativo y más seguro, que otro usado con mayor frecuencia (5, 16). Deporter D (2009), tras realizar una revisión de la literatura (9), concluye que para realizar carga inmediata es preciso una longitud de al menos $8 \mathrm{~mm}$ con un hueso de densidad mayor. Apunta que cuando no disponemos de una densidad favorable, excepcionalmente podemos recurrir a implantes de mayor diámetro $(4,8 \mathrm{~mm})$ en conjunción con técnicas modificadas en la colocación, como sería el uso de osteótomos de pequeño tamaño. Añade, que las características intrínsecas del implante a nivel del diseño, pueden minimizar la pérdida ósea. Asimismo, apunta a que los fallos en la prótesis se producen normalmente cuando varios implantes se hallan ferulizados por le rehabilitación. En contraste con lo expuesto, cuando la longitud de los implantes es menor de $8 \mathrm{~mm}$, se pueden usar junto con injertos de hueso medular o esponjoso. Es facti- ble colocarlos con una elevación de seno cuando hay al menos $3 \mathrm{~mm}$ de hueso y no requieren de ferulización aunque no está recomendado someterlos a carga inmediata. Felice $\mathrm{P}$ et al, en el año 2009, publican el artículo "Bone augmentation versus $5-\mathrm{mm}$ dental implants in posterior atrophic jaws. Four-month postloading results from a randomised controlled clinical trial" (15). Se trata de un ensayo clínico aleatorizado, que tiene como propósito evaluar si la colocación de implantes dentales cortos ( $5 \mathrm{~mm}$ ) con un diámetro de $6 \mathrm{~mm}$, puede ser una alternativa a los de mayor longitud $(10 \mathrm{~mm})$, en pacientes con atrofia mandibular. El estudio concluye que, cuando existe una altura residual de hueso de 5 a $7 \mathrm{~mm}$ sobre el canal mandibular, los implantes dentales cortos (5 $\mathrm{mm}$ ) quizás sean una opción preferible al aumento de hueso, ya que el tratamiento es más rápido, más barato y está asociado a una menor morbilidad. Cuando la altura del hueso es de 4 a $6 \mathrm{~mm}$ debajo del seno del seno maxilar, no está claro cuál es el procedimiento preferible. Por lo tanto, tomando todas estas conclusiones, que son las mismas que las obtenidas en ensayos clínicos controlados y aleatorizados previos $(14,43)$ así como una revisión sistemática (10) anterior, se puede sugerir como una técnica alternativa a corto plazo, mientras que a largo plazo deben estudiarse los resultados. Entre las principales limitaciones de esta investigación, fue el pequeño tamaño de la muestra. Los resultados preliminares de estos ensayos clínicos, controlados y aleatorizados (RCTs) sugieren que los implantes de longitudes de 7 a $8 \mathrm{~mm}$, pueden ser una mejor alternativa a los procedimientos de aumento óseo. También con alto grado de evidencia, Cannizzaro G, Felice P, Leone M, Viola P y Espósito M (2009) (5) nos presentan un ensayo clínico aleatorizado, donde en pacientes que presentan una atrofia maxilar superior con una altura residual de 3 a $6 \mathrm{~mm}$, que hace necesario la elevación lateral de seno para colocar implantes más largos (10 a $16 \mathrm{~mm}$ ). En estos casos, los implantes más cortos (de $8 \mathrm{~mm}$ ), quizás sea una elección preferible de tratamiento, ya que está asociado con una menor morbilidad. Este estudio también sugiere que es posible cargar tempranamente (en aproximadamente 7 semanas) implantes colocados en senos maxilares elevados y en los que se logró una estabilidad primaria suficiente durante la colocación. Este mismo año, Espósito M y cols. (10), publican una revisión sistemática, que da luz sobre la utilización de los implantes cortos "Los implantes cortos son más efectivos y causan menos complicaciones que los implantes convencionales colocados en el maxilar inferior reabsorbido aumentado con hueso de la cadera o sustitutos óseos (bloques de hueso de vaca). El hueso puede regenerarse en dirección horizontal y vertical mediante di- 
versas técnicas, pero no está claro qué técnicas son preferibles, y son frecuentes las complicaciones, en particular en el caso del aumento óseo en dirección vertical". (pag. 2). Posteriormente, este mismo grupo de trabajo (11) aporta una serie de conclusiones sobre el uso diversos métodos de elevación de seno, pero basadas en pocos ensayos, generalmente de bajo nivel de evidencia y teniendo seguimientos cortos. Por lo tanto deben ser vistos como un estudio preliminar e interpretados con gran cautela. Concluyen que todavía no está claro cuándo es necesario emplear procedimientos de elevación de seno. Los implantes cortos $(5 \mathrm{~mm})$ pueden ser cargado exitosamente en el hueso maxilar con una altura residual de 4 a $6 \mathrm{~mm}$, pero su pronóstico a largo plazo es desconocido. Elevar el suelo del seno en casos de 1 a $5 \mathrm{~mm}$ de altura residual de hueso, sin utilizar injerto óseo, puede ser suficiente el coágulo autógeno para regenerar hueso nuevo y permitir una rehabilitación protésica posterior. Los sustitutos de hueso (biomateriales) pueden ser utilizados exitosamente para reemplazar el hueso autógeno del paciente. Si la altura de hueso residual es de 3 a $6 \mathrm{~mm}$ una elevación atraumática de seno con la colocación de implantes de $8 \mathrm{~mm}$, puede ser un tratamiento apropiado y con menos complicaciones que una elevación traumática con ventana lateral y utilizando implantes de $10 \mathrm{~mm}$. Recientemente se han publicado trabajos donde los investigadores comparan la colocación de implantes cortos, con otras técnicas como el injerto con hueso bovino y no encuentran significada diferencia entre ambos. $(14,16)$. Concluyen que cuando la altura residual del hueso sobre el canal mandibular está entre 7 y $8 \mathrm{~mm}$, implantes de $7 \mathrm{~mm}$ quizás sean una opción preferible al aumento vertical mediante injerto, reduciendo el tiempo, los gastos y la morbilidad. Estos resultados preliminares al año, deben ser confirmados mediante un seguimiento de al menos 5 años. En el estudio realizado en 2010 por Quesada García (2010) (36), la longitud y el sistema de implantes no influyeron en la estabilidad, medida con el análisis de frecuencia de resonancia, a las 12 semanas tras la implantación. Sin embargo, el diámetro del implante sí influyó en la estabilidad del mismo. Así, los implantes de diámetro estrecho presentaron una estabilidad menor que el resto de los diámetros. Como apunta la autora, la longitud no ha mostrado influencia significativa en el valor ajustado del ISQ medida con RFA.

En cuanto a los estudios de análisis por elementos finitos (FEA: Finite Element Analysis) y aunque conociendo las limitaciones de este tipo de estudios, pueden aportar información de utilidad (18). Encontramos que un estudio de análisis biomecánico, concluye que au- mentando la longitud del implante, sólo puede aumentar la tasa de éxito hasta cierto punto. El uso de implantes cortos no ha sido recomendado extensamente porque se cree que las fuerzas oclusales, deben disiparse en un área mayor que aumenta con la longitud del implante. Aunque estudios biomecánicos (FEA) han mostrado que las fuerzas oclusales son distribuidas principalmente a la cresta del hueso, en lugar de hacerlo uniformemente a través de la totalidad del área entera de la interfase del implante-hueso. Estas fuerzas normalmente son bien toleradas por el hueso. Esto puede ser una razón de porqué la longitud del implante, no está relacionada linealmente con la estabilidad biomecánica (26). En la misma categoría de evaluación, nos encontramos con el realizado en 2010 por Hasan I, Heinemann F, Aitlahrach M y Bourauel C (22). En este trabajo, se presenta un análisis biomecánico del implante dental corto. Se trata de un análisis científico profundo del impacto mecánico y biomecánico de la longitud y el diámetro reducidos de los implantes. Una de las limitaciones del estudio fue, que la situación anatómica no es reproducida perfectamente. Pero en conclusión, los implantes cortos ofrecen varias ventajas quirúrgicas comparadas con implantes más largos. Los implantes más cortos, reducen el riesgo quirúrgico de perforación de seno o parestesia del nervio dentario inferior, con una reducción general en complicaciones quirúrgicas. Los implantes cortos y minis tienen ventajas clínicas significativas. Sin embargo, desde un punto de vista biomecánico, parece que la carga de hueso alrededor de los implantes cortos y minis, está aumentada en comparación con los de medida estándar. Adicionalmente, los resultados presentados muestran que hay un riesgo aumentado de sobrecarga y fractura para implantes minis, especialmente cuando se utilizan implantes de titanio grado 4. Por consiguiente, estos autores recomiendan el uso de los implantes cortos o minis, pero concluyen que un análisis biomecánico detallado de varias situaciones clínicas debe ser realizado para determinar el número necesario de implantes a colocar en estas situaciones clínicas. Añadiendo más parámetros en el análisis, nos encontramos con el estudio prospectivo de cohorte, realizado por Rossi F, Ricci E, Marchetti C, Lang NP y Botticelli D (2010) (41), donde aplica implantes cortos $(6 \mathrm{~mm})$ con una superficie moderadamente áspera con carga temprana (después de 6 semanas). Durante la cicatrización, obtuvo unas tasas altas de supervivencia del implante y una moderada pérdida de hueso después de 2 años de la carga. Parece esbozado ya, que decidirse terapéuticamente por la colocación de implantes cortos cuando existe indicación, es todo un "arte", y así lo denomina Neldam CA y Pinholt EM (31) 
en su estudio. El propósito fue evaluar sistemáticamente las publicaciones referentes a implantes dentales cortos, definidos con una longitud de $\leq 8 \mathrm{~mm}$ y colocados en el maxilar superior o en la mandíbula con especial referencia al tipo de implante, la tasa de supervivencia, la localización del implante y el tiempo de observación. El material y los métodos usados fue una búsqueda en Medline para identificar los estudios publicados entre 1992 y octubre 2009. Los artículos incluidos aportan datos al respecto (fecha, longitud, superficie, tiempo de observación, restauraciones únicas, tipos de implantes, etc). Se incluyeron 27 estudios: 15 prospectivos no randomizados y no controlados, 11 estudios retrospectivos no randomizados, una revisión y no hubo ningún ensayo clínico aleatorizado. Se encontraron pocos casos menores de $6 \mathrm{~mm}$ de longitud, y los más representados fueron fabricados por Straumann (representan 441 de 549). Los implantes de Branemark, $7 \mathrm{~mm}$ de largo, suponían 1.607 de 1.808. Los de Straumann, 8 $\mathrm{mm}$ de longitud, representaban 2.040 de 2.352. Los fracasos variaron entre $0-14,5 \%, 0-37,5 \%$ y $0-22,9 \%$ respectivamente para $6 \mathrm{~mm}, 7 \mathrm{~mm}$ y $8 \mathrm{~mm}$ de longitud. El período de análisis fue entre 2 meses y 14 años (algo que hace complicado la comparación de los datos). Los implantes con superficie mecanizada, mostraron generalmente, resultados menos favorables que aquellos que habían recibido tratamiento de superficie (lavado ácido o arenado). El estudio de la pérdida de implante, mostró una pauta general, concentrada durante la fase de cicatrización, la conexión al aditamento o durante el primer año de carga funcional. De nuevo, entre las conclusiones de esta revisión, destaca el hecho de la inexistencia de algún ensayo clínico aleatorizado sobre el uso de implantes cortos $(\leq 8 \mathrm{~mm}$ ) que aporte un nivel apropiado de evidencia. El mismo año, es publicada una revisión sistemática (25), realizada de forma manual y electrónica, obteniéndose un total respectivamente de 1.056 y 14.417 artículos. Se encontró que no existía diferencia estadísticamente significativa en la supervivencia entre corto $(\leq 8 \mathrm{y}<10 \mathrm{~mm})$ y convencional $(\geq 10 \mathrm{~mm}$ ) para implantes con tratamiento de superficie, colocados en pacientes total o parcialmente edéntulos. Concluyen que, dentro de las limitaciones de esta revisión sistemática, la colocación de implantes cortos de superficie rugosa no es una modalidad de tratamiento menos eficaz comparada con la colocación de implantes convencionales de superficie rugosa para el reemplazo de dientes perdidos en edéntulos totales y parciales. La última revisión sistemática publicada corresponde a Romeo et al (40). En ella, compara el uso de técnicas de aumento de hueso con el uso de implantes cortos, cuando existe reducción de las estructuras óseas. Como conclusión, la ma- yor parte de los estudios, aportaron datos sobre la existencia de diferencia en la tasa de supervivencia de los implantes, aunque esta diferencia no es significativa. Parece por tanto, que las tasas de menor éxito con implantes cortos, corresponden a estudios más antiguos frente a la literatura publicada más recientemente. Esta revisión destaca, además, la importancia de una buena planificación del tratamiento, como factor clave en el éxito, al usar implantes cortos. Nos orienta acerca de algunos de los parámetros que el clínico debe considerar a la hora de la rehabilitación:

1. El área a rehabilitar así como la calidad del hueso.

2. Longitud del implante.

3. Diámetro del implante

4. Tipo de implante y tratamiento de la superficie.

5. Corona a colocar y proporción corona-implante.

6. Tipo de prótesis.

7. La conexión a otros implantes (ferulización).

8. Carga oclusal/carga parafuncional.

9. Complicaciones protésicas.

$\mathrm{El}$ autor concluye diciendo que aunque en la literatura no hay estudios que analicen la supervivencia del implante corto, teniendo en cuenta cada uno de los puntos clave que inciden en su éxito o fracaso, puede asumirse que una planificación cuidadosa del tratamiento, dirigirá al clínico para obtener una rehabilitación con éxito. Controvertidamente, con respecto al tamaño de la corona, encontramos artículos que señalan que éste parece no influir en la supervivencia del implante corto (3). Más reciente aún, es este estudio experimental de Yang T-C, Maeda Y, Gonda T (2011) (47), donde se evaluó el comportamiento biomecánico de siete implantes cortos ferulizados, colocados en un bloque acrílico. Dos implantes de $7 \mathrm{~mm}$ longitud con el mismo diámetro $(3,8$, $4,4 \circ 5,0 \mathrm{~mm}$ ) fueron ferulizados juntos y se comparó con un implante de $4,4 \times 12 \mathrm{~mm}$, ejerciendo una carga oblicua de $50 \mathrm{~N}$ sobre las restauraciones. El estrés disminuyó apreciablemente con un aumento en el diámetro del implante. El estrés observado fue idénticos entre los 2 implantes ferulizados del mismo diámetro y estos 2 ferulizados con el implante largo, sugiriendo que ferulizando dos implantes cortos tiene la misma eficacia biomecánica que ferulizarlo a uno largo.

\section{Protocolización del uso terapéutico de los implantes cortos}

Las publicaciones más recientes dedicadas a implantes cortos han acentuado el uso de un protocolo quirúrgico adaptado, para obtener la estabilidad primaria adecuada. A este respecto Anitua E, Orive G, Aguirre 
JJ y Andrea I (1), se plantea como objetivo de su estudio evaluar las tasas a largo plazo de supervivencia de implantes cortos en áreas posteriores y analizar la influencia de factores diferentes en la supervivencia del implante. A través de un diseño retrospectivo de Cohorte, con una de 293 sujetos que recibieron 532 implantes cortos entre 2001 y 2004 . Aunque se trata de un estudio de un nivel de evidencia bajo, por las limitaciones en el control de la muestra, sobre todo, las tasas generales de supervivencia de implantes cortos fueron $99,2 \%$ y $98,7 \%$. Pero lo que sí se desprende es que el tratamiento con implantes cortos puede ser considerado seguro y previsible si es utilizado bajo protocolos clínicos estrictos. Pero no es hasta 2010 (2), que en su estudio retrospectivo de 9 años, nos presenta más claramente que respecto a los protocolos clínicos, son necesarios que sean estrictos para garantizar la seguridad clínica en la colocación de implantes cortos. En este artículo (estudio retrospectivo a largo plazo) que evalúa 1.287 implantes cortos, colocados en 661 sujetos, en 9 años, las tasas generales de supervivencia fueron $99,3 \%$ y $98,8 \%$. Pero como el propio autor indi$\mathrm{ca}$, no es el mejor tipo de estudio para determinar nuestra práctica clínica, debido entre otros a la pérdida posible de datos en el tiempo. Pero hacemos mención a este artículo, porque resulta ser uno de los que reúne el mayor número de implantes cortos consecutivamente colocados en la literatura. Adicionalmente, la seguridad y la funcionalidad a largo plazo de los implantes son documentadas bien, porque aproximadamente $50 \%$ del número total de implantes ha sido seguido de 48 a 102 meses Los estudios recientes muestran que los implantes pueden ser predecibles y que tienen tasas de éxito similares a las de implantes convencionales siempre que sean utilizados bajo una cuidadosa planificación de tratamiento y protocolos estrictos (4). Morand M. e Irinakis T (30), nos hace hincapié en la importancia de una adecuada planificación en el tratamiento, donde se incluya una comprensión exhaustiva de los materiales y los métodos disponibles al cirujano oral en estos tiempos.

\section{CONCLUSIONES}

A través de la revisión bibliográfica y descripción cronológica presentada en este trabajo, hemos hecho un recorrido por las distintas posturas de los autores respecto al uso de los implantes cortos y su evolución en los últimos años.

En primer lugar, apuntar el hecho de la dificultad en acotar la búsqueda en base al concepto "implante cor- to/short implants", ya que la definición en sí misma, ha evolucionado casi tanto, como los resultados obtenidos con estos. De esta manera, hablar de implante corto en 1990, suponía un concepto totalmente diferente a lo que actualmente entendemos como tal. Los artículos definían como corto, aquellos cuya longitud era $\leq 10 \mathrm{~mm}$, hablar de algo todavía más pequeño, era una auténtica "locura". Por otro lado, la inexistencia del tesauro "short dental implants", implica que los artículos que utilizan el concepto "short" como palabra clave, incluyen en su evaluación, diversos criterios de comportamiento en cuanto a la longitud del implante y no es comparable con los definidos como $\leq 8 \mathrm{~mm}$, algo que dificulta la tarea del clínico en la toma de decisiones terapéuticas, ya que las conclusiones obtenidas, varían en función de los criterios de selección de la longitud por parte de cada autor. En vista a los datos obtenidos, podemos afirmar que el uso de implantes cortos, es una práctica que en los últimos 6 años, ha adquirido solvencia científica, hasta tal punto que se puede recomendar, como una técnica de resultados previsibles, siempre y cuando se utilice como alternativa a otros métodos de tratamiento en caso de atrofia ósea y bajo protocolos estrictos. A partir de 2006 (y, sobre todo, en 2009), comienzan a aparecer artículos con un nivel de evidencia aceptable, como para recomendar su uso en determinadas circunstancias. Esto es, tras un estudio meticuloso del plan de tratamiento más adecuado y como alternativa a otras técnicas que pueden ser más cruentas, con mayor riesgo de fracaso y con mayor coste para el paciente. Estas técnicas de aumento de hueso requieren de procedimientos que suelen ser bastante complejos, y necesitan ser aplicados por cirujanos orales experimentados y hábiles, donde los pacientes suelen tener regímenes estrictos de control postoperatorio y a menudo aparecen complicaciones. Sin recurrir a las técnicas de injerto óseo, no hemos encontrado en la bibliografía, alguna evidencia acerca de cual es la cresta residual mínima necesaria, para la colocación de implantes cortos versus implantes cigomáticos. La primera cuestión que el implantólogo debería plantearse, es cuáles son los beneficios que conlleva para el paciente el aplicar dicho procedimiento a diferencia de otros de aumento óseo. Por lo tanto, estos beneficios deben ser sopesados con cautela frente al riesgo de sufrir complicaciones inherentes a la cirugía avanzada. En cuanto a los porcentajes de éxito y fracaso en comparación con los implantes de longitud estándar, nos encontramos con un problema continuamente referenciado en la literatura: la inexistencia de ensayos clínicos aleatorizados que comparen distintas variables. Es necesario un ensayo que controle to- 
das las variables posibles, multiplicándose de manera idéntica y que solo evalúe, el efecto directo de la longitud del implante sobre el éxito a corto, medio y largo plazo. Es crucial el aislamiento de la influencia exacta de la longitud, de manera individualizada sobre otros parámetros. Esto no es el caso para la mayoría de los ensayos controlados aleatorios encontrados (ECAs), dado que comparan una combinación de diferentes características de superficie, formas, dimensiones, pureza del titanio y colocados con diferentes protocolos quirúrgicos (sumergido versus no sumergido, carga temprana o inmediata, uso de diferentes tipos de injertos, etc.).

Los cambios en las decisiones terapéuticas, se implementan en base a recomendaciones científicas fruto de las investigaciones en la materia. Respecto al uso de implantes cortos, se ha hecho en base a "sospechas" de evidencia hasta que aparecieron los primeros ECAS y revisiones sistemáticas, ya que hasta entonces, solo existían en la bibliografía, un gran número de casos clínicos documentados, sobre todo en estudios longitudinales, que habían demostrado éxito o al menos, no existencia de diferencia en la tasa de fracaso con respecto a los de longitudes estándares. Lo que sí vislumbra la literatura, es la necesidad de que exista un protocolo clínico y prostético para garantizar el éxito en la rehabilitación.

A destacar el hecho de que el uso de implantes cortos, es una práctica habitual desde hace bastantes años, pero en comparación con la nutrida producción de otros temas de interés científico, no ha gozado de la suficiente atención, como objeto de estudio con rigor científico, muy a pesar, de que un gran número de pacientes, podrían beneficiarse de las conclusiones obtenidas de la Ciencia.

\section{BIBLIOGRAFÍA}

1. Anitua E, Orive G, Aguirre JJ, Andrea I. Five-Year Clinical Evaluation of Short Dental Implants Placed in Posterior Areas: A Retrospective Study. J. Periodontol 2008;79:428.

2. Anitua E, Orive G. Short Implants in Maxillae and Mandibles: A Retrospective Study With 1 to 8 Years of Follow-Up. J Periodontol 2010;81:819-26.

3. Birdi H, Schulte J, Kovacs A, Weed M, Chuang S, Crownto-Implant Ratios of Short-Length Implants Journal of Oral Implantology Six/2010.Vol. XXXVI/No.
4. Cabosmalón, NL, Buera B, Martínez JA., Celemín A. Revista Internacional de Prótesis Estomatológica. Edición Hispanoamericana, 2010 OCT-DIC; 12 (4).

5. Cannizzaro G, Felice P, Leone M, Viola P, Esposito M. Early loading of implants in the atrophic posterior maxilla: lateral sinus lift with autogenous bone and BioOss versus crestal mini-sinus lift and $8 \mathrm{~mm}$ implants. A randomized controlled clinical trial. Eur J Oral Implantol 2009;2:25-38.

6. Cannizzaro G, Leone M, Torchio C, Viola P, Esposito M. Immediate versus early loading of 7-mm-long flaplessplaced single implants: a split-mouth randomised controlled clinical trial. Eur J Oral Implantol. 2008Winter; l(4):277-92.

7. Chung DM, Tae-Ju Oh, Lee J, Misch CE, Hom-Lay Wang. Factors Affecting Late Implant Bone Loss: A Retrospective Analysis. Int J Oral Maxillofac Implants 2007;22:117-26 controlled clinical trial of up to 4 months after loading. Eur J Oral Implantol 2009;2:7-20.

8. Das Neves FD, Fones D, Bernardes SR, do Prado CJ, Neto AJ. Short implants - an analysis of longitudinal studies. Int J Oral Maxillofac Implants 2006;21:86-93.

9. Deporter D. Dental Implant Design and Optimal Treatment Outcomes. Int J Periodontics Restorative Dent 2009;29:625-33.

10. Espósito M, Grusovin MG, Felice P, Karatzopoulos G, Worthington $\mathrm{H}$, Coulthard P. Intervenciones para reemplazar los dientes perdidos: técnicas de aumento óseo horizontal y vertical para el tratamiento con implantes dentales (Revision Cochrane traducida). En: Biblioteca Cochrane Plus 2009 Número 4. Oxford: Update Software Ltd. Disponible en: http://www.update-software.com. (Traducida de The Cochrane Library, 2009 Issue 4 Art no. CD003607. Chichester, UK: John Wiley \& Sons, Ltd.).

11. Esposito M, Grusovin MG, Rees J, Karasoulos D, Felice P, Alissa R, Worthington $\mathrm{H}$, Coulthard P. Effectiveness of sinus lift procedures for dental implant rehabilitation: a Cochrane systematic Review. Eur J Oral Implantol 2010; 3(1):7-26.

1 lb. Esposito M, Worthington HV, Coulthard P. Interventions for replacing missing teeth: dental implants in zygomatic bone for the rehabilitation of the severely deficient edentulous maxilla. Cochrane Database of Systematic Reviews 2005, Issue 4. Art. No.: CD004151. DOI: 10.1002/14651858.CD004151.pub2. 
12. Feldman S, Boitel N, Weng D, Kohles SS, Stach RM. Fiveyear survival distributions of short-length $(10 \mathrm{~mm}$ or less) machined surfaced and Osseotite implants. Clin Implant Dent Relat Res 2004; 6:16-23.

13. Felice P, Cannizzaro G, Checchi V, Marchetti C, Pellegrino G, Censi P, Esposito M. Vertical bone augmentation versus 7-mm-long implants in posterior atrophic mandibles. Results of a randomised controlled clinical trial of up to 4 months after loading. Eur J Oral Implantol 2009;2(1)7-20.

14. Felice P, Cannizzaro G, Checchi V, Pellegrino G, Censi P, Esposito M. Vertical bone augmentation versus 7-mmlong dental implants in posterior atrophic mandibles. Results of a randomised controlled clinical trial of up to 4 months after loading. Eur J Oral Implantol. 2009 Spring; $2(1): 7-20$.

15. Felice P, Checchi V, Pistilli R, Scarano A, Pellegrino G, Esposito M. Bone augmentation versus $5-\mathrm{mm}$ dental implants in posterior atrophic jaws. Four-month postloading results from a randomised controlled clinical trial. Eur J Oral Implantol 2009;2(4) 267-81.

16. Felice P, Pellegrino G, Checchi L, Pistilli R, EspositoM. Vertical augmentation with interpositional blocks of anorganic bovine bone vs. 7-mm-long implants in posterior mandibles: 1-year results of a randomized clinical trial. Clin. Oral Impl. Res. 21, 2010;1394-403.

17. Ferrigno N, Laureti M, Fanali S. Dental implants placement in conjunction with osteotome sinus floor elevation: a 12-year life-table analysis from a prospective study on 588 ITIs implants. Clin. Oral Impl. Res. 17,2006;194-205

18. Georgiopoulos B, Kalioras K, Provatidis C, Manda M, Koidis P. The Effects Of Implant Length And Diameter Prior To And After Osseointegration: A 2-D Finite Element Analysis. Journal of Oral Implantology. Five/ 2007. Vol. XXXIII/No.

19. Goené R, Bianchesi C, Hüerzeler M, et al. Performance of short implants in partial restorations: 3-year followup of Osseotite implants. Implant Dent 2005; 14:274-80.

20. Grunder U, Polizzi G, Goené R, et al. A 3-year prospective multicenter follow-up on the immediate and delayedimmediate placement of implants. Int J Oral Maxillofac Implants 1999;14:210-6.

21. Hagi D, Deporter DA, Pilliar RM, Arenovich T. A Targeted Review of Study Outcomes With Short ( $<7 \mathrm{~mm}$ ) Endosseous
Dental Implants Placed in Partially Edentulous Patients". J Periodontol 2004;75:798-804.

22. Hasan I, Heinemann F, Aitlahrach M, Bourauel C. Biomechanical finite element analysis of small diameter and short dental implant. Biomed Tech 2010;55:341-50.

23. Jae-Hoon Lee, Val Frias, Keun-Woo Lee, Robert F.Wright. Effect of implant size and shape on implant success rates: A literature review. J Prosthet Dent 2005;94:37781 .

24. Jiménez Fábrega J, Casas Hernández A. Diseño macroscópico de los implantes y su relación con la biomecánica. En: Sociedad española de periodoncia y osteointegración, eds. Manual Sepa de Periodoncia y terapéutica de implantes. Madrid: Panamericana; 2005. p. 285-97.

25. Kotsovilis S, Fourmousis I, Karoussis IK,Bamia C. "A Systematic Review and Meta-Analysis on the Effect of Implant Length on the Survival of Rough-Surface Dental Implants". J Periodontol, November 2009.

26. Lee JH, Frias V, Lee KW,Wright RB. Effect of implant size and shape on implant success rates: $A$ literature review. J Prosthet Dent 2005;94:377-81.

27. Meyer U, Vollmer D, Runte C, Bourauel C, Joos U. Bone loading pattern around implants in average and atrophic edentulous maxillae: A finite-element analysis. J Craniomaxillofac Surg 2001;29:100-5.

28. Misch CE, Steigenga J, Barboza E, Misch-Dietsh F, Cianciola LJ, Kazor C. Short Dental Implants in Posterior Partial Edentulism: A Multicenter Retrospective 6Year Case Series Study. J Periodontol 2006;77:1340-7.

29. Misch CE. Short dental implants: a literature review and rationale for use. Dent Today 2005;24(8):64-6,68. 31.

30. Morand M, Irinakis T. The Challenge of implant therapy in the posterior maxilla: Providing a rationale for the use of short implants. Journal of Oral Implantology. Vol. XXXIII/No. Five/2007.

31. Neldam CA, Pinholt EM. State of the Art of Short Dental Implants: A Systematic Review of the Literaturecid_303 1..11. Clinical Implant Dentistry and Related Research, 2010.Volume 2010 DOI: 10.1111/j.1708-8208.2010.00303.x

32. O'Sullivan D, Sennerby L, Meredith N. Measurements comparing theinitial stability if five designs of dental 
implants: A human cadáver study. Clin Implants Dent Reí Res. 2000;2:85-92.

33. Petrie CS, Williams JL. Comparative evaluation of implant designs: influence of diameter, length, and taper on strains in the alveolar crest. A three-dimensional finiteelement analysis. Clin. Oral Impl. Res. 16, 2005;486-94

34. Pierrisnard L, Renouard F, Renault P, Barquins M. Influence of Implant Length and Bicortical Anchorage on Implant Stress Distribution. Clinical Implant Dentistry and Related Research, 2003Volume 5, Number 4.

35. Porter JA, von Fraunhofer JA. Success or failure of dental implants? A literature review with treatment. Gen Dent. 2005 Nov-Dec;53(6) :423-32; quiz 433, 446.

36. Quesada García, MJ. Tesis doctoral: Factores que influyen en la estabilidad de los implantes dentales medida con el análisis de frecuencia de resonancia. Editorial Universidad de Granada. Abril 2010.

37. Renouard F, Nisand D. "Impact of implant length and diameter on survival rates". Clin Oral Implants Res 2006;17(suppl 2) :35-51.

38. Renouard F, Nisand D. Short implants in the severely resorbed maxilla: a 2-year retrospective clinical study. Clin Implant Dent Relat Res 2005;7(Suppl 1) :S104-S1 10.

39. Rokni S, Todescan R, Watson P, Pharoah M, Adegbembo AO, Deporter D. An assessment of crown-to-root ratios with short sintered porous-surfaced implants supporting prostheses in partially edentulous patients. Int J Oral Maxillofac Implants 2005;20:69-76.

40. Romeo E, Bivio A, Mosca D, Scanferla M, Ghisolfi M, Storelli S. The use of short dental implants in clinical practice: literature review. Minerva Stomatologica. 2010 January-February;59(1-2):23-31.

41. Rossi F, Ricci E, Marchetti C, Lang NP, Botticelli D. Early loading of single crowns supported by 6-mm-long implants with a moderately rough surface: a prospective 2-year follow-up cohort study. Clin. Oral Impl. Res. 21, 2010;937-43.

42. Snauwaert K, Duyck J, van Steenberghe D, Quirynen M, Naert I. Time dependent failure rate and marginal bone loss of implant supported prostheses: a 15-year followup study. Clin Oral Investig 2000;4:13-20.

43. Stellingsma K, Raghoebar GM, Meijer HJ, Stegenga B. The extremely resorbed mandible: a comparative prospective study of 2-year results with 3 treatment strategies. Int J Oral Maxillofac Implants 2004;19:563577.

44. Tada S, Stegaroíu R, Kitamura E et al. Influence of implant desing and bone quality on stress/strain distribution in bone around implants: a dimensional fínite element analysis. Int J Oral Maxillofac Implante. 2003;18:35768.

45. Van Steenberghe D, Lekholm U, Bolender C, Folmer T, Henry P, Herrmann I. Applicability of osseointegrated oral implants in the rehabilitation of partial edentulism: a prospective multicenter study on 588 fixtures. Int J Oral Maxillofac Implants 1990;5:272-81.

46. Winkler S, Morris HF, Ochi S. Implant survival to 36 months as related to length and diameter. Ann Periodontol. 2000;5:22-31.

47. Yang T-C, Maeda Y, Gonda T. Biomechanical Rationale for Short Implants in Splinted Restorations: An In Vitro Study. Int J Prosthodont 2011;24:1302.

\section{CORRESPONDENCIA}

Rodrigo Azañón Hernández Urb. Mirador de Rolando Fase II. Casa-8. 18011 Granada

\section{Correo electrónico: razanon@gr.epes.es}

